\title{
Analysis of the Dependence of Stock Risk Based on Copula Theory
}

\author{
Qi Li, Guangming Deng*, Xin Tan \\ College of Science, Guilin University of Technology, Guilin, China \\ Email: *804616492@qq.com
}

How to cite this paper: Li, Q., Deng, G. M., \& Tan, X. (2019). Analysis of the Dependence of Stock Risk Based on Copula Theory. Journal of Financial Risk Management, 8, 224-231. https://doi.org/10.4236/jfrm.2019.84015

Received: October 25, 2019

Accepted: November 25, 2019

Published: November 28, 2019

Copyright ( 2019 by author(s) and Scientific Research Publishing Inc. This work is licensed under the Creative Commons Attribution International License (CC BY 4.0).

http://creativecommons.org/licenses/by/4.0/

(c) (i) Open Access

\begin{abstract}
The rapid development of the economy emphasizes the importance of financial risk. Financial risk analysis can help people understand finance more deeply and reduce the loss of profits. This paper is about the dependence on stocks, which is very important for analyzing the dependence structure of stock market and the portfolio risk of investment market. The experimental data are the daily closing price data of shares of Midea Group and Gree Electric. Copula theory is used to fit the daily return data of Gree Electric and Midea Group. By establishing the correlation structure model of the stock market, the daily return data of Gree Electric and Midea Group are better simulated.
\end{abstract}

\section{Keywords}

Stock Risk, Dependence, Copula Function

\section{Introduction}

The rapid development of the economy emphasizes the importance of financial risk. Longin \& Solnik (2001) proposed that testing the hypothesis that international equity market correlation increases in volatile times is a difficult exercise and misleading results have often been reported in the past because of a spurious relationship between correlation and volatility. As a result of the complex relationship between stock markets and within the market, the fluctuation of one risk is likely to cause the fluctuation of other risk factors. The efficiency of the Chinese wheat and soybean futures markets is studied by Wang \& He (2005). The exact definition of Copula function is given by Nelsen (2006). Zhang proposed that copula technique is a kind of comparatively new method of financial risk analysis, whose core is to connect the co-distribution of many random variances with their fringe distributions. Copula function is very important in the 
analysis of market risk. Hammoudeh et al. (2014) examine the recent trends in dependence structure between the fast-growing commodity markets and the stock markets in China by using copula functions.

This paper selected daily closing price data of Gree Electric and Midea Group shares and analyzed the daily return of the two stocks by copula function.

\section{Copula Function}

Nelsen (2006) proposed that Copula function is a join function that connects the joint distribution function $F\left(x_{1}, x_{2}, \cdots, x_{N}\right)$ of random vectors $X_{1}, X_{2}, \cdots, X_{N}$ with their respective edge distribution functions $F_{X_{1}}\left(x_{1}\right), F_{X_{2}}\left(x_{2}\right), \cdots, F_{X_{N}}\left(x_{N}\right)$. That is, the function $C\left(u_{1}, u_{2}, \cdots, u_{N}\right)$, making

$$
F\left(x_{1}, x_{2}, \cdots, x_{N}\right)=C\left[F_{X_{1}}\left(x_{1}\right), F_{X_{2}}\left(x_{2}\right), \cdots, F_{X_{N}}\left(x_{N}\right)\right] .
$$

\subsection{Properties of Binary Distributed Copula Function}

- $C(u, v)$ is incremental for all its variables;

- for any $u$ and $v(u, v \in[0,1]), C(u, 0)=C(0, v)=0, C(u, 1)=u$, $C(1, v)=v$;

- For any $u_{1}, u_{2}, v_{1}$ and $v_{2}$, if $0 \leq u_{1} \leq u_{2} \leq 1$ and $0 \leq v_{1} \leq v_{2} \leq 1$, then $C\left(u_{2}, v_{2}\right)-C\left(u_{2}, v_{1}\right)-C\left(u_{1}, v_{2}\right)+C\left(u_{1}, v_{1}\right) \geq 0$;

- For any $u_{1}, u_{2}, v_{1}, v_{2}$, if $u_{1} \in[0,1], u_{2} \in[0,1], v_{1} \in[0,1]$ and $v_{2} \in[0,1]$, then $\left|C\left(u_{2}, v_{2}\right)-C\left(u_{1}, v_{1}\right)\right| \leq\left|u_{2}-u_{1}\right|+\left|v_{2}-v_{1}\right|$

- If $U$ and $V$ are independent and obey the uniform distribution on $[0,1]$, then $C(u, v)=u v$.

\subsection{Gaussian Copula Function and $t$-Copula Function}

Two-dimensional Gaussian Copula function:

$$
C^{G a}(u, v, \rho)=\int_{-\infty}^{\Phi^{-1}(u)} \int_{-\infty}^{\Phi^{-1}(v)} \frac{1}{2 \pi \sqrt{1-\rho^{2}}} \exp \left(-\frac{s^{2}-2 \rho s t+t^{2}}{2\left(1-\rho^{2}\right)}\right) d s d t,
$$

where $\rho$ is a dependent parameter, $-1 \leq \rho \leq 1, \Phi^{-1}$ and $\Phi^{-1}$ are normal distribution function and inverse function respectively.

Two-dimensional $t$-Copula function:

$$
C^{G a}(u, v, \rho, k)=\int_{-\infty}^{t_{k}^{-1}(u)} \int_{-\infty}^{t_{k}^{-1}(v)} \frac{1}{2 \pi \sqrt{1-\rho^{2}}} \exp \left(-\frac{s^{2}-2 \rho s t+t^{2}}{k\left(1-\rho^{2}\right)}\right)^{-(k+2) / 2} \mathrm{~d} \mathrm{~d} t,
$$

where the linear correlation coefficient between variables is $-1 \leq \rho \leq 1$, the degree of freedom is $k$.

Gaussian Copula function and $t$-Copula function have symmetrical tails, but Gaussian Copula function can't capture the asymmetric tail correlation between random variables. The $t$-Copula function has a thick tail, which is very sensitive to the changes of random variables distributed in dimensions, and can better capture the tail correlation changes. 


\subsection{Construction of Copula Model}

The steps to build copula model are as follows:

1) According to the daily closing price data, the daily return rate of stock is calculated and its statistical characteristics are described.

2) The kernel density estimation method is used to construct the edge distribution, so as to fit the variation characteristics and distribution characteristics of random variables.

3) Catching the dependent structure between random variables by Gaussian Copula function and $t$-Copula function.

\section{Empirical Analysis}

\subsection{Sample Selection}

This paper selected the daily closing price data of shares of Midea Group and Gree Electric from July 14, 2007 to October 17, 2019, to study the internal relationship between two stock markets. The data comes from Oriental Fortune net. According to the daily closing price, the daily return of the stock is obtained, and its frequency histogram is given below. It can be seen from Figure 1 and Figure 2 that the distribution of the two-time series is relatively uniform, showing the characteristics of peak and thick tail, but the two-time series are not normal distribution.

\subsection{Nonparametric Estimation}

Kernel density estimation method is selected for nonparametric estimation. It is

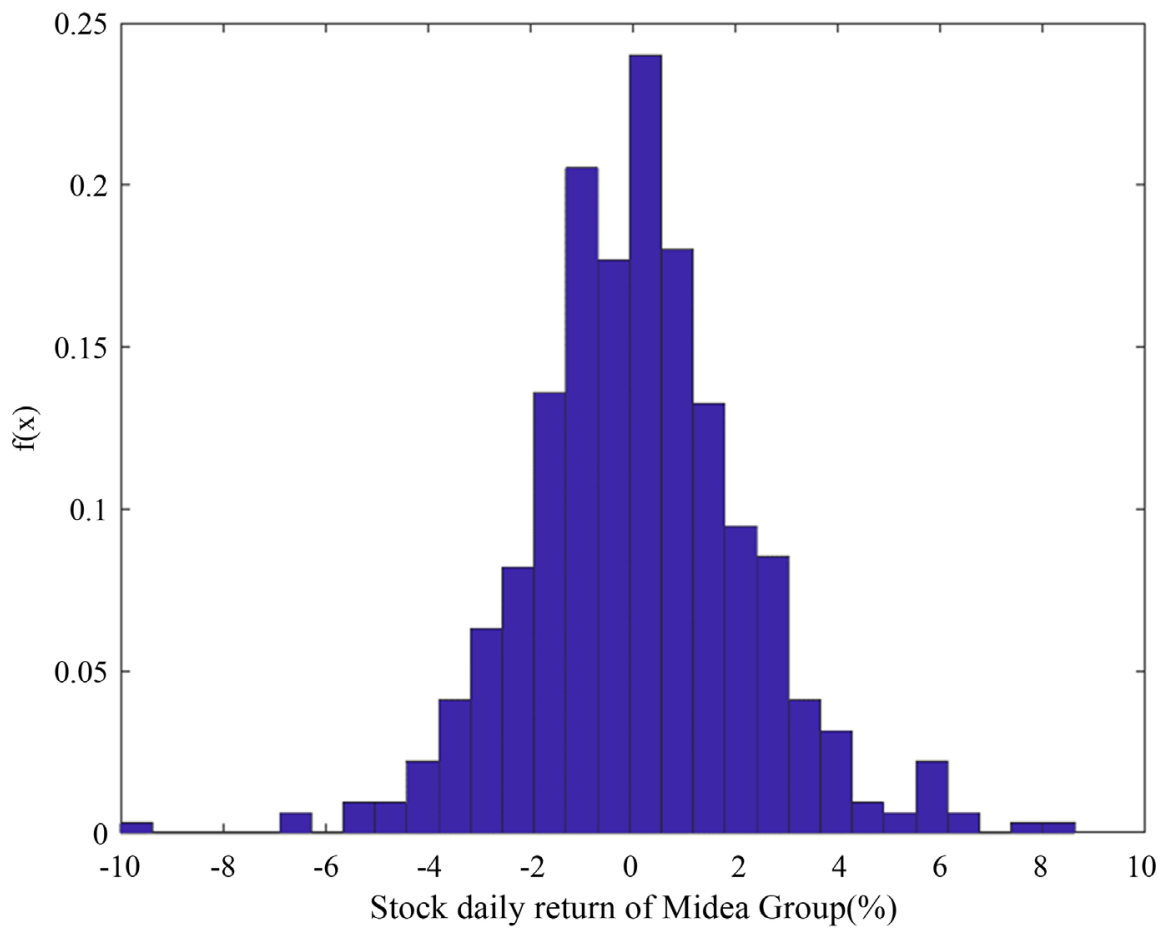

Figure 1. Frequency histogram of daily return of Midea Group. 


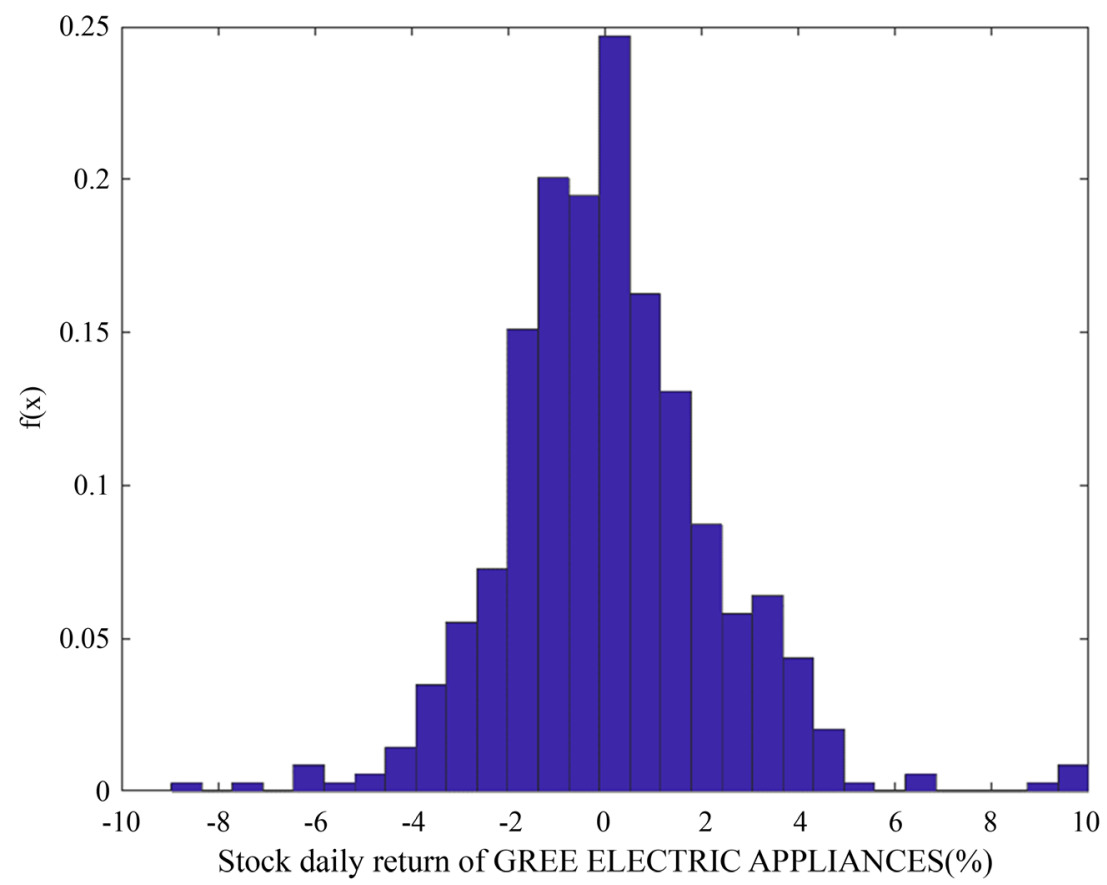

Figure 2. Frequency histogram of daily return of Gree Electric.

defined as: If the random sample of a single variable $x$ is $x_{1}, x_{2}, \cdots, x_{n}$, the nonparametric density estimation method of the probability density distribution function $f(x)$ of $x$ is defined as:

$$
f_{n}(x)=\frac{1}{n h_{n}} \sum_{i=1}^{n} K\left(\frac{x-X_{i}}{h_{n}}\right),
$$

where $K()$ is kernel function.

Table 1 gives the statistics of daily return, such as mean, variance, skewness and kurtosis. The two-time series are not normal distribution since their kurtosis is large than 3.

This paper chooses Gaussian function as the kernel function since the time series of stock return has the characteristics of peak and thick tail.

It can be seen from Figure 3 and Figure 4 that the empirical distribution function of the stock daily return of Midea Group and Gree Electric is basically consistent with the estimation of the nonparametric kernel distribution, and the nonparametric kernel density estimation can well estimate the overall distribution of the sample.

\subsection{Selection of Copula Function}

Figure 5 shows the binary frequency histogram. Obviously, the daily return data of the tail are similar, so the tail of the two series is basically symmetrical. So $(u, v)$ has a tail symmetric joint density function. This paper chooses the binary Gaussian Copula function and the binary $t$-Copula function to describe the correlation of the original sample data. The estimated value of linear correlation parameter $\rho$ of Gaussian Copula function is: 
Table 1. Statistics of daily return.

\begin{tabular}{cccccc}
\hline Company & $\mathrm{N}$ & Mean & Variance & Skewness & Kurtosis \\
\hline Midea Group & 510 & 0.0798 & 4.7646 & 0.1148 & 4.6121 \\
Gree Electric & 510 & 0.1386 & 4.9937 & 0.4588 & 5.7310 \\
\hline
\end{tabular}

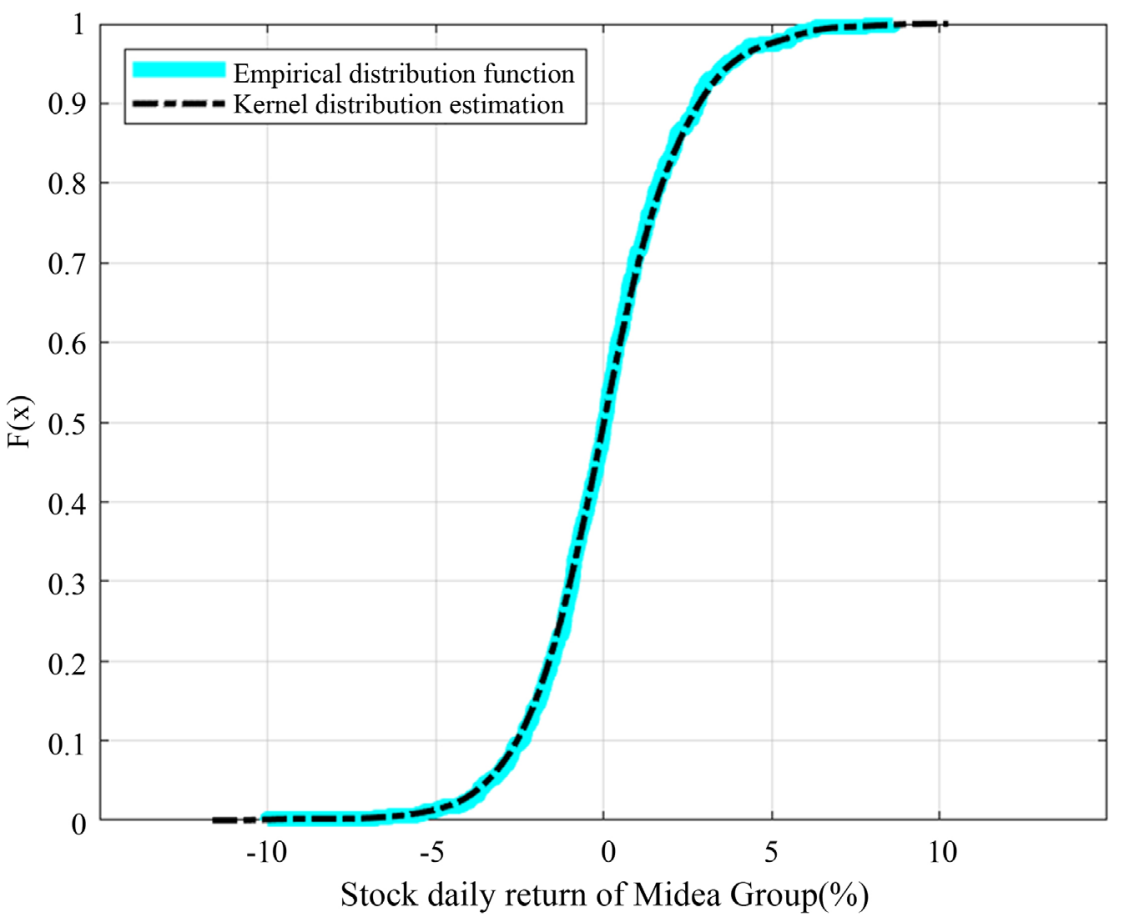

Figure 3. Empirical distribution function and kernel distribution of Midea Group.

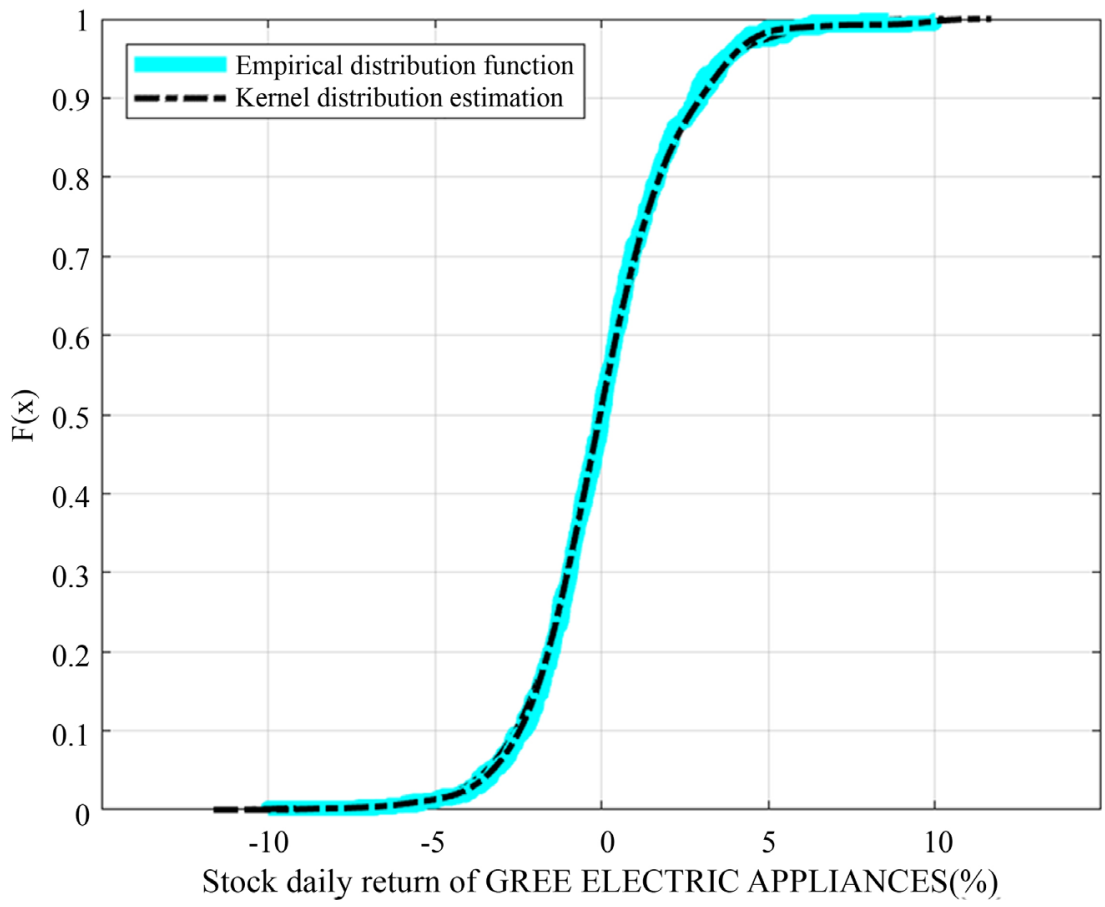

Figure 4. Empirical distribution function and kernel distribution of Gree Electric. 

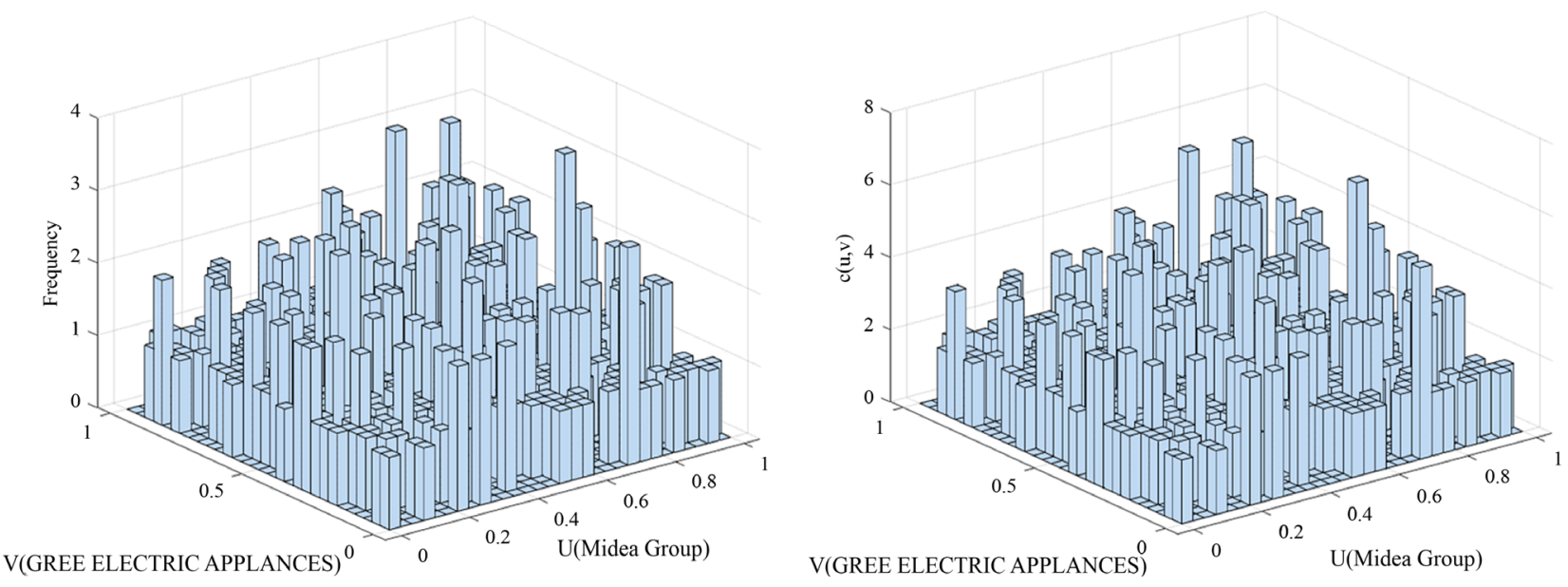

Figure 5. Statistical graph of binary Copula function.

$$
\hat{\rho}_{1}=\left[\begin{array}{ll}
1.0000 & 0.0654 \\
0.0654 & 1.0000
\end{array}\right] .
$$

The estimated value of linear correlation parameter $\rho$ and degree of freedom $k$ of $t$-Copula function is

$$
\hat{\rho}_{2}=\left[\begin{array}{ll}
1.0000 & 0.0701 \\
0.0701 & 1.0000
\end{array}\right],
$$

and the estimated value of degree of freedom $k$ of $t$-Copula function is $\hat{k}=2.2496 \mathrm{e}+06$.

Figure 6 and Figure 7 show the density distribution functions and distribution functions of the binary Gaussian Copula function and the binary $t$-Copula function. It can be seen that density distribution functions of binary Gaussian Copula function and the binary $t$-Copula function are similar.

\subsection{Model Evaluation}

In order to verify the goodness of fit of binary Gaussian Copula function and the binary $t$-Copula function, this paper selected the method of squared Euclidean distance. The smaller the square Euclidean distance is, the better the fitting effect of Copula function is. The square Euclidean distance can be calculated by the following formula:

$$
d^{2}=\sum_{i=1}^{n}\left|\hat{C}_{n}\left(u_{i}, v_{i}\right)-\hat{C}\left(u_{i}, v_{i}\right)\right|,
$$

where $\hat{C}_{n}\left(u_{i}, v_{i}\right)$ is an empirical Copula function. The fitting effect of binary Gaussian Copula function and the binary $t$-Copula function are similar since the square Euclidean distances between empirical Copula function and the two Copula functions.

\section{Conclusion}

The main contribution of this paper is to select the daily closing price data of shares of Midea Group and Gree Electric as the research object, use copula function 

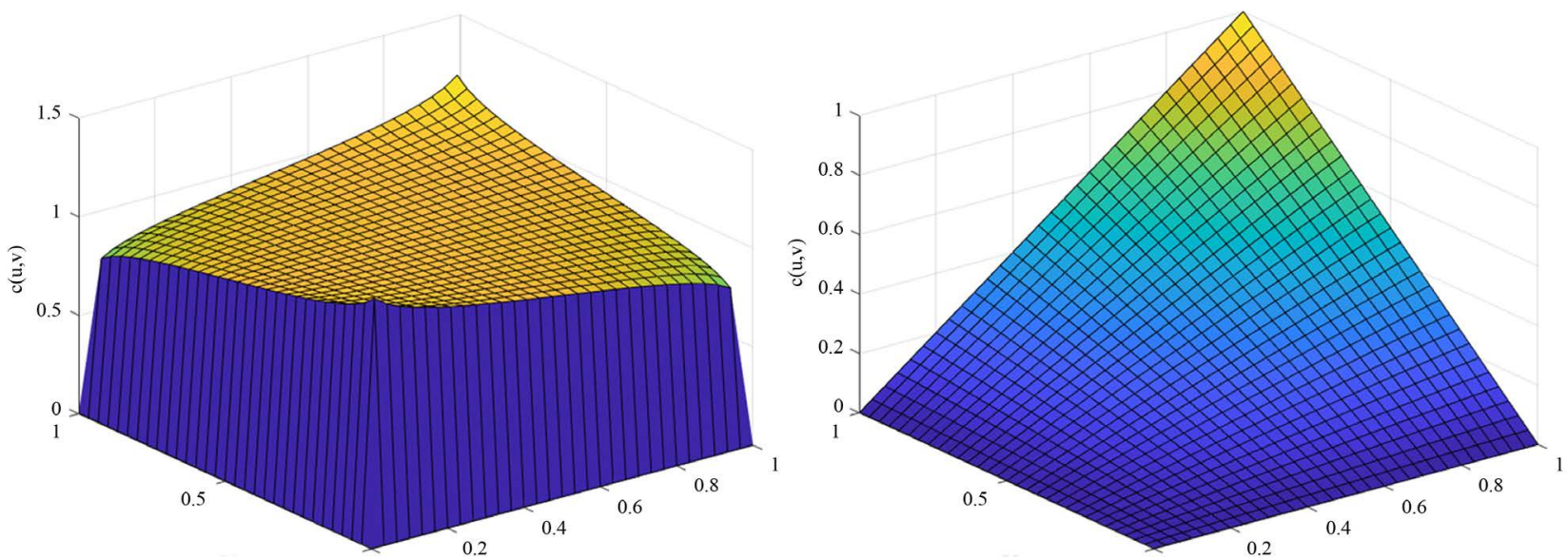

Figure 6. The density distribution function and distribution function of the binary Gaussian Copula function.
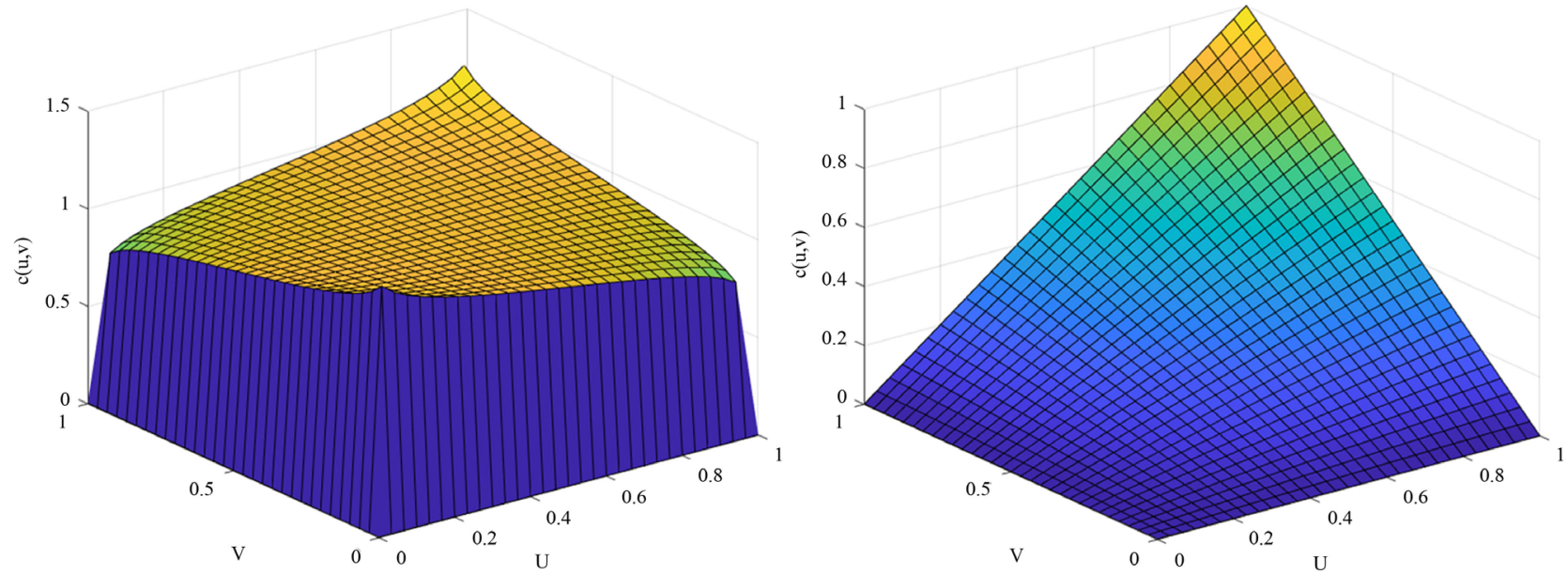

Figure 7. The density distribution function and distribution function of the binary $t$-Copula function.

to establish the model, and analyze the daily return of stock. The results show that the binary Gaussian Copula function and the binary $t$-Copula function can simulate the daily return data of the stock market very well. Copula function is very important in the analysis of market risk. Copula function can be used to better simulate financial data and decompose financial risks, so as to reduce the impact of financial risks. This paper only selects the data of two stocks in China, which are not very representative. In order to better study the role of Copula function in financial risk analysis, it is necessary to select more representative data.

\section{Acknowledgements}

This paper is financially supported by National Natural Science Foundation of China (NSFC) under Grant number 71963008.

\section{Conflicts of Interest}

The authors declare no conflicts of interest regarding the publication of this paper. 


\section{References}

Hammoudeh, S., Nguyen, D. K., Reboredo, J. C., \& Wen, X. (2014). Dependence of Stock and Commodity Futures Markets in China: Implications for Portfolio Investment. Emerging Markets Review, 21, 183-200. https://doi.org/10.1016/j.ememar.2014.09.002

Longin, F., \& Solnik, B. (2001). Extreme Correlation of International Equity Markets. The Journal of Finance, 56, 649-676. https://doi.org/10.1111/0022-1082.00340

Nelsen, R. B. (2006). An Introduction to Copulas. An Introduction to Copulas.

Wang, H. H., \& Ke, B. (2005). Efficiency Tests of Agricultural Commodity Futures Markets in China. Australian Journal of Agricultural \& Resource Economics, 49, 125-141. https://doi.org/10.1111/j.1467-8489.2005.00283.x 